\title{
A Fluoroscopic X-Ray Registration Process for Three-Dimensional Surgical Navigation
}

\author{
Robert Grzeszczuk $^{1}$, Shao Chin ${ }^{1}$, Rebecca Fahrig ${ }^{2}$, Hamid Abassi ${ }^{1}$, \\ Hilary Holz ${ }^{1}$, Daniel Kim ${ }^{1}$, John Adler ${ }^{1}$, Ramin Shahidi ${ }^{1}$ \\ ${ }^{1}$ Image Guidance Laboratories, Stanford University \\ ${ }^{2}$ Department of Radiology, Stanford University
}

\begin{abstract}
We describe a system involving a computer-instrumented fluoroscope for the purpose of 3D navigation and guidance using pre-operative diagnostic scans as a reference. The goal of the project is to devise a computerassisted tool that will improve the accuracy, reduce risk, minimize the invasiveness, and shorten the time it takes to perform a variety of neurosurgical and orthopedic procedures of the spine. For this purpose we propose an apparatus that will track surgical tools and localize them with respect to the patient's anatomy and pre-operative 3D diagnostic scans using intraoperative fluoroscopy for in situ registration. The resulting system leverages equipment already commonly available in the Operating Room (OR), allowing us to provide important new functionality that is free of many current limitations, while keeping costs contained.
\end{abstract}

\section{Introduction}

Computer-assisted spine surgery is an active area and numerous systems, both commercial and research, have been described in the literature. Most of these systems provide similar functionality and methodology. The majority employ optical trackers for the purpose of registration and localization. Typically, the vertebral body of interest is fully exposed intra-operatively and a small number of distinct anatomical landmarks are digitized for the purpose of coarse registration. Subsequently, a larger number of points are digitized on the surface of the vertebrae to refine the registration with a surface matching technique. The procedure is often cumbersome, time consuming, and of limited accuracy. This is mainly due to difficulties in identifying characteristic anatomical landmarks in a reproducible fashion (particularly in older patients and pathologically changed bone structures) and inherent inaccuracies of surface matching techniques. While dynamic reference frames (DRFs) are commonly used to monitor target movement, any safeguarding against DRF misregistration requires the entire process, including the laborious manual digitization part, to be repeated. This gets even more cumbersome in procedures involving multiple vertebrae (e.g., cage placements) requiring multiple DRFs to be maintained. Finally, aforementioned techniques cannot be applied in the context of percutaneous procedures, because they rely on the target structure being directly visible to the optical tracking device.

Recently, several academic researchers [1] and commercial vendors (e.g., FluoroNav, Medtronic, Minneapolis, MN,USA) began experimenting with fluoroscopy as an intraoperative imaging modality. The relative low cost and pervasiveness of C-Arm devices in modern ORs can explain this increased interest. Most of these attempts 
focus on improving conventional 2D navigation techniques via tracking of the C-Arm and re-projecting pre-operative CT data onto multiple planes. Such techniques are helpful in lowering the amount of ionizing radiation delivered to the patient and the OR staff during free-hand navigation and also in providing more information to the surgeon about the relative position of the surgical tools with respect to the patient's anatomy. However, they essentially automate and streamline the current workflow and rely on the surgeon's ability to create a complex spatial model mentally. In contrast, our approach employs stereoscopic registration in order to relate patient's anatomy to pre-operative diagnostic scans in $3 \mathrm{D}$.

\section{Materials and Methods}

\subsection{Overview}

A mobile fluoroscopic device (C-Arm, GE, Milwaukee, WI, USA) is used for instrumented intra-operative free hand imaging of skeletal anatomy. The position and orientation of its camera is tracked by an optoelectronic device (Optotrack, Northern Digital, Waterloo, Ontario, Canada). NTSC video output of the X-ray camera as well as its position and orientation are processed by an SGI 320 (SGI, Mountain View, CA, USA) NT workstation. The image data is used by the system to register a preoperative CT data set $(512 \times 512130$ slices, $1.0 \mathrm{~mm}$ thick) to the patient's reference frame. Tracking data is used to monitor the relative position changes of the camera with respect to the patient during free-hand navigation.

During the course of the procedure, the system operator performs an initial registration of the patient's anatomy to the pre-operative CT study by taking two protocoled fluoroscopic views (e.g., AP and lateral) of the target vertebrae. These images are used to compute the $\mathrm{C}$-Arm-to-CT registration using a fully automatic technique described in detail below. As a result, we obtain the position and orientation of the C-Arm's camera in the reference frame of the CT study. We can then use this information, together with the position and orientation of the camera in the tracker reference frame to follow the future relative motions of the camera, assuming static system. Misregistration, due to either patient movement or system error, can be detected at any time by comparing the predicted DDR to the actual fluoroscopic image, at which point the objects can be re-registered within seconds. A surgical tool visible in at least two fluoroscopic views can be back-projected into the CT reference frame using standard stereoscopic techniques well known in the computer vision community. Alternatively, we can track a surgical tool externally (e.g., using the optical tracking device already employed, or a robotic interface) to facilitate a variety of procedure types.

\subsection{Intrinsic Camera Calibration}

Before the system can be used effectively, it needs to be calibrated to eliminate imaging distortions and extract parameters required to characterize the imaging system. During the process of intrinsic camera calibration we determine the set of parameters that characterize the internal geometry of our imaging system. All of these parameters are well documented in the computer vision literature [2]: effective focal 
length (i.e., the exact distance from the X-ray source to the image plane), focal spot (i.e., the exact place where the optical axis pierces the image plane), magnification factors, and pincushion distortions. It is also well understood that many of C-Arm's intrinsic parameters vary with orientation. For example, the focal length and focal spot will be different for vertical and horizontal positioning, due to mechanical sag of the arm. Similarly, the pincushion distortion depends on the orientation of the device with respect to the Earth's magnetic field, and external source of EMFs. For these reasons we fix a calibration jig to the face of the image intensifier, in order to adaptively calibrate the system every time an image is acquired. The intrinsic parameters thus produced are used during the registration phase.

\subsection{Extrinsic Camera Calibration and Registration}

Once the intrinsic camera parameters are measured, we can proceed to register our imaging system to the patient. The problem can be posed as follows: given a CT study of the relevant anatomy and a fluoroscopic image of the same anatomy, find the position and orientation of the camera in the reference frame of the CT study that produced the image. For this purpose, we adopted a technique used by a commercial frameless image-guided radiosurgery system (Cyberknife, Accuray Inc., Sunnyvale, CA, USA) [3]. In this approach, the system hypothesizes about the camera's actual six degrees of freedom (DOF) position by introducing a virtual camera and computing a simulated radiograph (Digitally Reconstructed Radiograph, or DRR). The radiograph represents the actual patient position. If the DRR matches the radiograph exactly, the virtual camera position identifies the actual camera position and thus the registration is found, otherwise another hypothesis is formed. In practice, the accuracy of such basic radiograph-to-DRR registration method can be substantially improved if two or more views of the same anatomy can be used. In this scenario, we will assume that the relative positions of the cameras are known (e.g., from an optical tracking device), so instead of finding two or more independent camera positions [4], we can reformulate our problem as that of finding the pose of CT study with respect to the rigid configuration of multiple cameras. In essence, we can view this registration process as an extrinsic calibration of an abstract imaging system consisting of multiple, rigidly mounted cameras.

The radiograph-to-DRR registration problem has three parts. First, we need to develop the means of exploring the range of possible patient positions to be represented in the DRRs (i.e., hypothesis formation strategy), second, identify those elements in the images (image features) that most effectively capture information about the patient pose, then finally, third, use a comparison statistic or cost function for the two sets of image feature data to indicate when the best match has been achieved.

There are three fundamental ways of generating hypotheses for possible patient placement. One approach, called matched filter area correlation, generates a single reference image from a DRR representing the desired patient position from the camera point of view. This reference image, or part thereof, is shifted and rotated relative to the acquired image until the best fit is achieved, in a manner of a sliding window matched filter. A second approach, referred to here as interpolative area correlation, 
consists of calculating a set of reference DRRs that samples a full range of possible patient positions and orientations, and then making an interpolative comparison of the acquired fluoroscopic image with each of the DRRs. Using two cameras, either of the aforementioned methods can accurately measure all threes translational and one rotational degree of freedom, providing there is no out-of-plane degrees of freedom [6]. A third method consists of interactively re-projecting DRRs while perturbing the pose of the patient image in the CT study, until a DRR is made that matches the fluoroscopic image. The iterative re-projection technique can accurately measure all six DOFs and is the approach we have taken here.

Comparison of the DRRs and acquired radiographs is a problem in pattern recognition. The sets of image data used to compare two images are called feature vectors. The most primitive feature vector is simply the complete set of pixel grayscale values, where each pixel's brightness is the magnitude of a vector component. More sophisticated feature vectors are usually sought to emphasize the important large-scale structure in the image and minimize the extraneous information and noise. Formal systems of feature extraction involve recasting the original grayscale feature vector on an orthogonal system of eigenvectors that relate to large-scale patterns that are not necessarily physical structures. Heuristic feature vectors identify the positions and shapes of physical edges, boundaries, and other discernible structures.

The DRR and radiograph feature vectors $\mathbf{A}$ and $\mathbf{B}$ are compared using a similarity statistic or cost function. This can be simply the cross-correlation coefficient $r=\mathbf{A} \bullet$ $\mathbf{B}=\cos \theta$, where $\mathbf{A}$ and $\mathbf{B}$ have been normalized to unit length. (The vectors can be centered on their means before normalization, which gives Pearson's correlation coefficient.) A more general and flexible comparison can be made with the chisquared statistic: $\chi^{2}=\Sigma\left(\mathrm{A}_{\mathrm{i}}-\mathrm{B}_{\mathrm{i}}\right)^{2} / \mathrm{w}_{\mathrm{i}}{ }^{2}$, where each vector component is weighted according to both its reliability and usefulness, and the vectors are not necessarily normalized. When the vectors are normalized, and all vector components carry equal weight, $\chi^{2}$ is proportional to $1-r$.

\section{Results}

For fiducial-based registration, the fluoroscope image contrast is expanded and then thresholded to highlight the fiducial shadows. The image-plane coordinates of the fiducials are automatically extracted using one of three methods: (1) A Sobel edge filter is convolved across the image to further enhance the fiducials, the image is thresholded again, and then $\mathrm{x}$ and $\mathrm{y}$ moments are computed in the neighborhood of the bright fiducial edge features; (2) a matched filter representing the shadow of the fiducial is convolved across the image and the convolution maxima are isolated by thresholding; (3) if spherical fiducials have been used, a circular Hough transform is applied to the image, resulting in a bright maximum at the center of each fiducial shadow. Registration of skeletal landmarks is accomplished by edge-filtering the fluoroscope and DRR reference images and then locating the points where the anatomical edges intersect line segments at fixed positions in the images. These points make up the feature vectors for the radiographs and DRRs. With eight to ten 
feature points in each fluoroscope view, the translational registration can again achieve $\pm 0.2 \mathrm{~mm}$ and \pm 0.5 degrees precision.
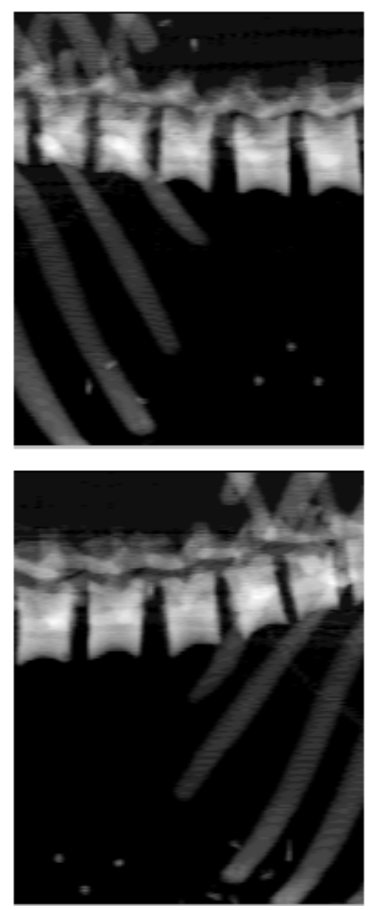
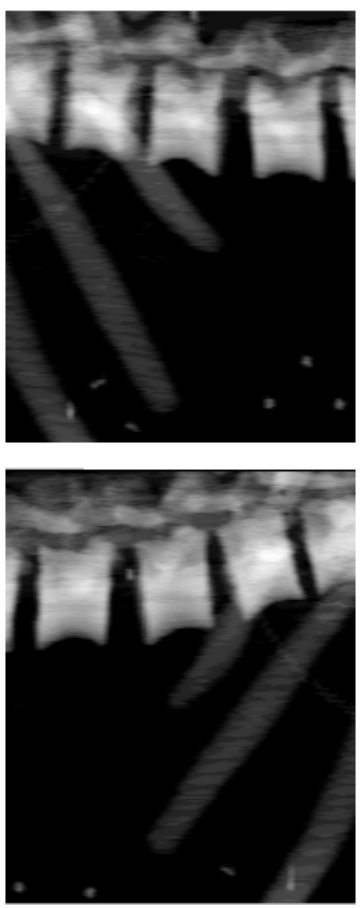
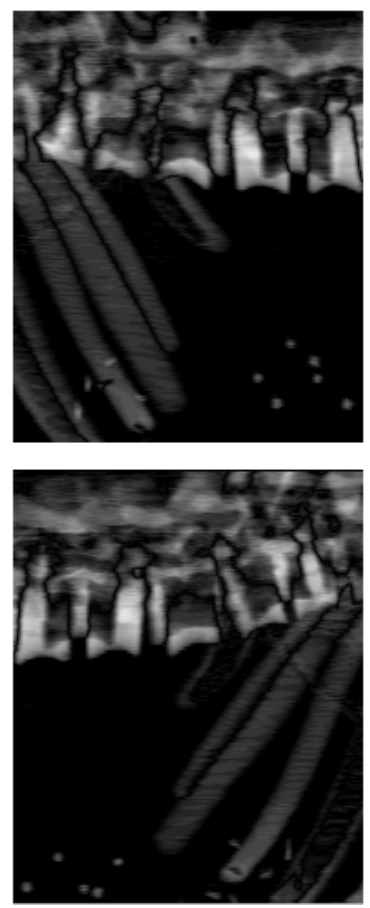

Figure 1. Registration in progress for a rigid torso phantom. Top row shows data for camera position A, the bottom for position B. Left column shows test DRRs, center the actual fluoroscopic images, while the right one shows the results of the correlation (in a mis-registered configuration).

If the fluoroscope images have a pixel pitch of $1.0 \mathrm{~mm}$, the position of a $2-3 \mathrm{~mm}$ diameter spherical fiducial can be found with a precision of $\pm 0.2 \mathrm{~mm}$. This yields a translational registration precision of $0.2 \mathrm{~mm}$ or better. The rotational precision depends on the fiducial spacing and the angle of projection of the fiducial configuration in the fluoroscope image. For fiducials spaced $25 \mathrm{~mm}$ apart a typical projection angle will resolve out-of-plane rotations with a precision of \pm 0.5 degrees.

\section{Discussion}

The main factors differentiating our solution from that of others include: selecting fluoroscopy for the in situ imaging technique, using stereo photogrammetry to extract $3 \mathrm{D}$ information from projective images, as well as using a robust, precise and practical registration method. Unlike all currently available 3D spinal navigation packages, which require full exposure of the vertebral body for the sake of registration and realtime optical tracking, our method employs fluoroscopic imaging and registration using percutaneously implanted markers or skeletal anatomy as a minimally invasive 
approach. This allows us to help guide surgical tools using pre-operative 3D diagnostic scan. While our method does not permit a real-time DRF for the sake of target movement monitoring, periodic re-registration is much more practical than in conventional approaches: misregistration can be detected and eliminated by simply reacquiring two new fluoroscopic images and running a fairly automatic procedure that requires little or no intervention by the operator. The registration technique has been adopted from Accuray's Cyberknife radiation treatment methodology, which has been shown to register either fiducial markers or skeletal anatomy with sub-millimeter and sub-degree precision in all six degrees of freedom and computation efficiency leading to a time scale of approximately one second to complete the entire registration process. Additional proprietary improvements in digital re-projection techniques using off-the-shelf computer graphics hardware will further enhance the robustness, accuracy, and performance of the registration method.

Similarly, our registration method coupled with more sophisticated visualization and biomechanical modeling techniques can potentially be generalized to handle non-rigid deformations resulting from inter-vertebral displacement. This would allow us to apply our methodology in clinical scenarios that involve more than a single vertebral body, without cumbersome patient fixation or making assumptions about unchanged pose between the time of the scan and intra-operative positioning. The ability to intraoperatively register articulated, deformable spinal anatomy in near real-time and with high accuracy would be a critical improvement over existing systems.

An important differentiating factor is our ability to track surgical tools with respect to the patient's anatomy as defined by a pre-operative diagnostic scan. Unlike traditional approaches where optical tracking is used to follow the surgical tools, we employ fluoroscopy for both registration and tool tracking. This permits us to apply our system in the context of minimally invasive percuteaneous procedures where the tool may not be exposed and visible to the tracking CCD camera. This is particularly advantageous for flexible and/or articulated effectors, which cannot be tracked optically. Finally, an additional benefit of our approach is more effective use of the fluoroscope with less exposure to ionizing radiation on the part of the patient as well as the surgeon, because instrumented fluoroscopy can be used in a more controlled manner than during conventional free hand imaging.

\section{References}

1. R. Hofstetter, M. Slomczynski, M. Sati and L.-P. Nolte, CAS, 4:65-76, 1999.

2. R.Y. Tsai, "An Efficient and Accurate Camera Calibration Technique for 3D Machine Vision", Proceedings of IEEE Conference on Computer Vision and Pattern Recognition, Miami Beach, FL, 1986, pages 364-374.

3. M. J. Murphy, "An automatic six-degree-of-freedom image registration algorithm form image-guided frameless stereotaxic radiosurgery," in Medical Physics 24(6), June 1997.

4. J. Weese, G.P. Penny, T.M. Buzug, C. Fassnacht and C. Lorenz "2D/3D registration of pre-operative CT images and intra-operative X-ray projections for image guided surgery," in CARS97, H.U. Lemke, M.W.Vannier and K Inamura ed., pages 833-838, 1997.

5. M. Roth, C. Brack, R. Burgkart, A. Zcopf, H. Gotte and A. Schwiekard "Multi-view contourless registration of bone structures using single calibrated X-ray fluoroscope," CARS99, pages 756-761, 1999. 